\title{
BCCN4
}

\section{BRAZILIAN SPLITTING TEST - EXPERIMENTAL AND NUMERICAL ANALYSIS}

\author{
Marcelo De Simone $^{(1)}{ }^{(2)}$, Lourdes M. S. Souza ${ }^{(2)}$ and Deane Roehl (1) (2)
}

(1) Department of Civil and Environmental Engineering, Pontifícia Universidade Católica do Rio de Janeiro, Brazil

(2) Instituto Tecgraf, Pontifícia Universidade Católica do Rio de Janeiro, Brazil

https://doi.org/10.21452/bccm4.2018.02.04

\begin{abstract}
The Brazilian splitting test is commonly used in order to estimate the tensile strength of brittle materials such as rocks and concretes. This test is performed by compressing a specimen disc in the direction of its diameter. Splitting tensile stresses are induced perpendicularly to the direction of the applied compressive load. Even though this test has been established for decades, standards, such as the ASTM D3967 - 16, still suggest numerous different test configurations, which may lead to different results.

In the present study, Brazilian Splitting Tests were conducted on Indiana Limestone discs of $54 \mathrm{~mm}$ diameter and $25 \mathrm{~mm}$ thickness. The discs were placed on flat platens. Digital Image Correlation (DIC) was used in order to obtain the strain fields developed during the tests. This technique allows the measurement of displacement/strain fields without contact.

The development of the stress fields and the crack formation were investigated by numerical models using the Finite Element Method (FEM). Two FEM models were carried out: Model I simulated a concentrated applied load (scenario I) while Model II considered a distributed load (scenario II). The model from scenario I resulted in a strain field on the sample surface similar to the one observed by DIC during the tests with flat platens. There was a higher tensile strain in the horizontal direction near the point of applied load. This indicates that, when flat platens are used, a concentrated load is applied, as expected.

Although this study is still a work in progress, the preliminary results indicate that, when load is applied using flat platens, the crack initiates at the load application point, while crack initiates in the center of the sample when load is distributed.
\end{abstract}

\section{INTRODUCTION}

Direct tensile tests are difficult to be performed on brittle materials, such as rocks and concretes. Alternatively, the Brazilian splitting test (BST), also known as indirect tensile test, is commonly used in order to estimate the tensile strength of such materials. The BST is performed by compressing the sample in the direction of its diameter. Splitting tensile stresses are induced perpendicularly to the direction of the applied compressive load, inducing tensile 
fracture. Even though this test has been established for decades, various studies and standards, such as the ASTM D3967-16 [1], suggest numerous different test configurations, which may lead to different results. Li \& Wong [2] presented a comprehensive review on the test, including the different loading configurations that have been used. Generally, it is assumed that the disc specimen is loaded uniformly and that the crack forms in the center of the specimen. However, this is not always observed in the laboratory and the validity of the test has been debated.

In order to improve the understanding of measurements from the laboratory tests, numerical and analytical solutions have been employed. Both Finite Element Method (FEM) and Discrete Element Method (DEM) have been used in the literature. Han et al. [3] presented a methodology to measure the elastic properties ( $E$ and $v$ ) using the BST. In addition, the authors compared the results for stress and strain from a finite element model with an analytical solution. Using discrete element models, Xu et al. [4] and Ma \& Huang [5] studied the sample behavior during the Brazilian Splitting Test.

In the present paper, the preliminary results for an experimental, analytical and numerical study on the Brazilian splitting test is presented. Indiana Limestone samples were submitted to the BST with digital image correlation (DIC) analysis to evaluate the strain development. The development of the stress fields was further investigated by FEM and analytical models.

\section{EXPERIMENTAL PROCEDURE}

In this study, Brazilian Splitting Tests were conducted on four discs of Indiana Limestone of $54 \mathrm{~mm}$ diameter and $25 \mathrm{~mm}$ thickness. The tests were conducted on a MTS testing machine, model 810 with load capacity of $250 \mathrm{kN}$, under displacement control of the actuator at a rate of $0.02 \mathrm{~mm} / \mathrm{min}$. The discs were placed on flat platens. The splitting tensile strength was calculated by the equation ASTM D3967-16, , [1]:

$\sigma_{t}=\frac{2 P}{\pi t D}$

where $\sigma_{\mathrm{t}}$ is the splitting tensile strength (in $\mathrm{MPa}$ ), $\mathrm{P}$ is the maximum applied load (in $\mathrm{N}$ ), $\mathrm{t}$ and $\mathrm{D}$ are the thickness and diameter of the specimen (in $\mathrm{mm}$ ), respectively.

Digital Image Correlation (DIC) method was used in order to obtain the strain fields developed during the tests. This technique allows the measurement of displacement/strain fields without contact. Two CCD cameras with 5MP (Point Grey GRAS-50S5M) with resolution of 2448 x 2028 pixels and high resolution lenses type Tamron A031 (AF28-200mm $\mathrm{F} / 3.8-5.6)$ were used and the pictures were taken at the rate of $1 \mathrm{fps}$. The software VIC SNAP and VIC-3D 2010, both from Correlated Solutions Inc., were used for image acquisition and processing. The test apparatus is shown in Figure 1. For the DIC method, the specimens were painted white with black speckles. 


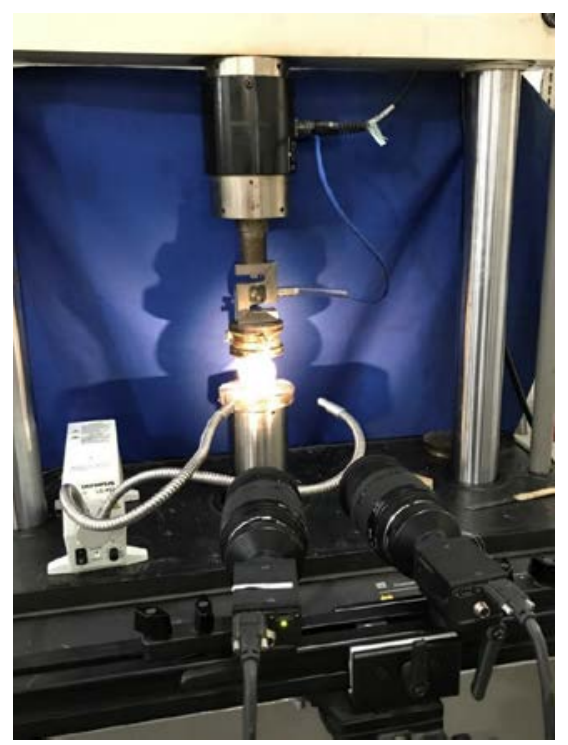

Figure 1: System used for the DIC in the Brazilian test

\section{FINITE ELEMENT METHOD MODEL}

The development of the stress fields and the crack formation were investigated by numerical models using the Finite Element Method (FEM). Two different models were carried out using the FEM software Abaqus. Model I simulated a concentrated applied load (scenario I) while Model II considered a distributed load (scenario II). In these models, the linear elastic assumption was adopted for simplicity. Based on Walton et al. [6], the Young's Modulus was considered to be 24.6 GPa while the Poisson's coefficient was assumed as 0.16.The load (P), the diameter (D) and the thickness (t) were obtained from the laboratory tests.

The models simulate a plane stress condition, with quadratic elements and reduced integration. The only difference between these two models is the applied load, as presented in Figure 2.
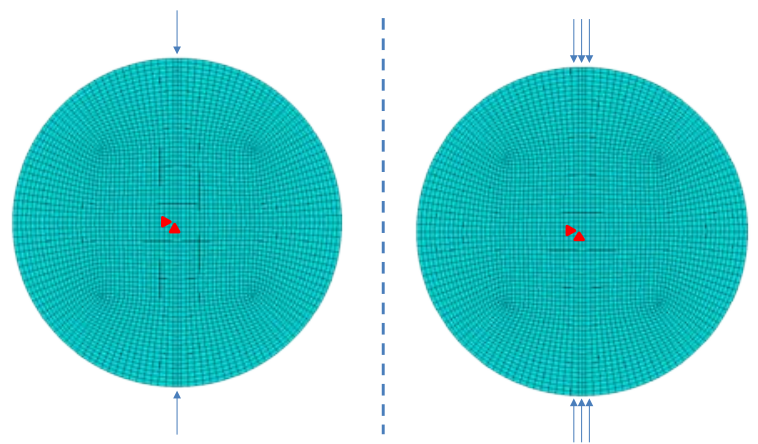

a) b)

Figure 2: a) Scenario 1 with concentrated load and b) Scenario2 with distributed load

These models are obviously simplified, as they are developed only to compare the results from the strain field from DIC with the finite element. Therefore, the main objective of the FEM modelling in the present paper is to observe the strain (horizontal (x) and vertical (y) directions) field around the sample. 


\section{ANALYTICAL SOLUTION}

The strain and stress fields over the sample surface were also studied using two analytical solutions. The first (Analytical 1) was presented by Jianhong et al. [7] and considers a concentrated applied load (Figure 3a).

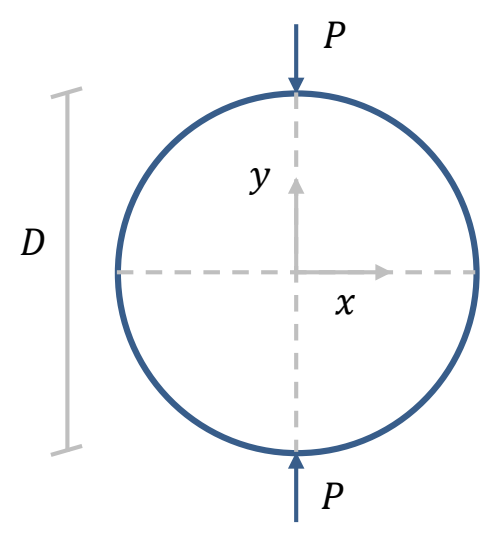

a)

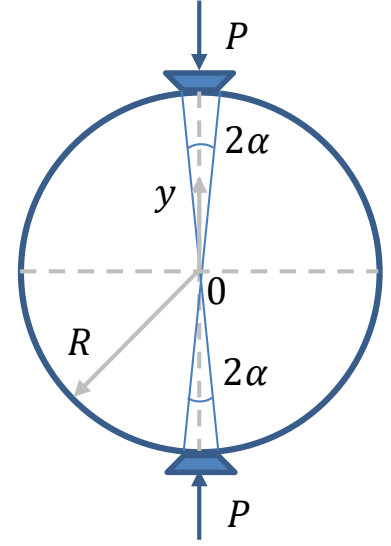

b)

Figure 3: Load application assumption for the analytical solution presented by a) Jianhong et al. [7] and b) Li \& Wong [2].

In this solution, equation (1) presents the horizontal stress and equation (2) the vertical stress in any point of the model.

$$
\begin{aligned}
& S_{x x}(x, y)=\frac{2 P}{\pi t}\left\{\frac{\left[\left(\frac{D}{2}\right)-y\right] x^{2}}{\left[\left(\left(\frac{D}{2}\right)-y\right)^{2}+x^{2}\right]^{2}}+\frac{\left[\left(\frac{D}{2}\right)+y\right] x^{2}}{\left[\left(\left(\frac{D}{2}\right)+y\right)^{2}+x^{2}\right]^{2}}-\frac{1}{D}\right\} \\
& S_{y y}(x, y)=\frac{2 P}{\pi t}\left\{\frac{\left[\left(\frac{D}{2}\right)-y\right]^{3}}{\left[\left(\left(\frac{D}{2}\right)-y\right)^{2}+x^{2}\right]^{2}}+\frac{\left[\left(\frac{D}{2}\right)+y\right]^{3}}{\left[\left(\left(\frac{D}{2}\right)+y\right)^{2}+x^{2}\right]^{2}}-\frac{1}{D}\right\}
\end{aligned}
$$

The second analytical equation (Analytical 2) considers a distributed load in a small arc length, $2 \alpha$ (Figure 3b). This solution was presented by Li \& Wong [2], where the stresses in the horizontal and vertical directions are evaluated along the direction of the applied load. Those stresses are presented in equation (3) and (4) for the horizontal and vertical directions, respectively.

$$
\begin{aligned}
& S_{x x}(y)=\frac{P}{\pi R t \alpha}\left\{\frac{\left[1-\left(\frac{y}{R}\right)^{2}\right] \sin (2 \alpha)}{1-2\left(\frac{y}{R}\right)^{2} \cos (2 \alpha)+\left(\frac{y}{R}\right)^{4}}-\tan ^{-1}\left[\frac{1+\left(\frac{y}{R}\right)^{2}}{1-\left(\frac{y}{R}\right)^{2}} \tan (\alpha)\right]\right\} \\
& S_{y y}(y)=-\frac{P}{\pi R \operatorname{ta} \alpha}\left\{\frac{\left[1-\left(\frac{y}{R}\right)^{2}\right] \sin (2 \alpha)}{1-2\left(\frac{y}{R}\right)^{2} \cos (2 \alpha)+\left(\frac{y}{R}\right)^{4}}+\tan ^{-1}\left[\frac{1+\left(\frac{y}{R}\right)^{2}}{1-\left(\frac{y}{R}\right)^{2}} \tan (\alpha)\right]\right\} \\
& 7
\end{aligned}
$$




\section{RESULTS AND DISCUSSION}

The Indiana Limestone specimens had average splitting strength of $1.43 \pm 0.12 \mathrm{MPa}$, with average maximum applied load of $3.05 \pm 0.20 \mathrm{kN}$. After the sample fails under tensile stress, a crack propagates in the middle of the sample in the same direction of the applied load. Using digital image correlation (DIC), the picture from the sample right before failure was used in order to get the strain field over the sample surface. Figure 4 presents the strain field in the horizontal and vertical directions of IL2 with $54 \mathrm{~mm}$.

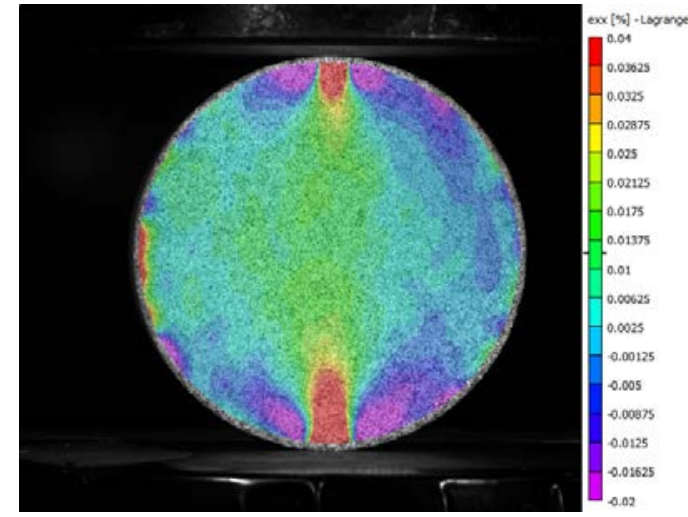

a)

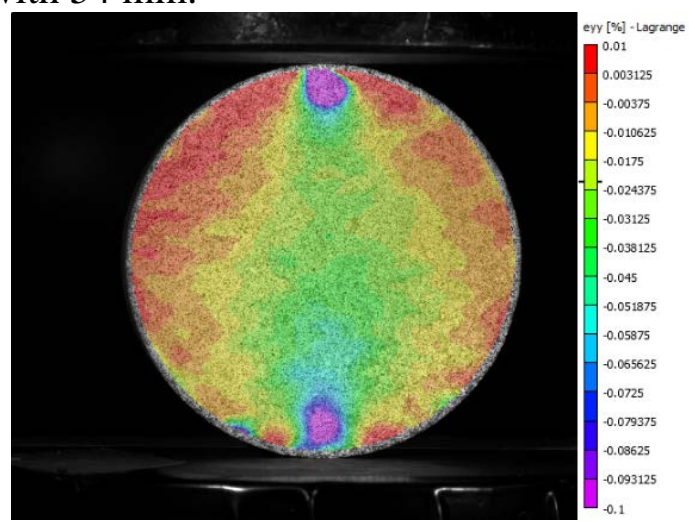

b)

Figure 4: Strain field in a) horizontal and b) vertical directions of sample IL2 with $54 \mathrm{~mm}$ in diameter.

From the DIC results, stress concentration is clear around the applied load points, both in horizontal stress (tensile) and in vertical stress (compressive). This observation is similar to the assumption from Model 1, presented in sections 3 and 4. For each sample, the DIC result showed a similar trend. This trend shows higher strains starting on the point of applied load and propagating to the center of the sample. This is expected as the plate is in direct contact with the sample. In order to avoid this stress concentration, some techniques are discussed in the ASTM D3967-16 [1].

Before comparing numerical, analytical and laboratory results, Model 1 and Model 2 are compared, considering both numerical and analytical solutions. The result for the vertical stress along the direction of the applied load is presented in Figure 5 for the numerical and analytical solutions, while the result for the horizontal direction is described in Figure 6.

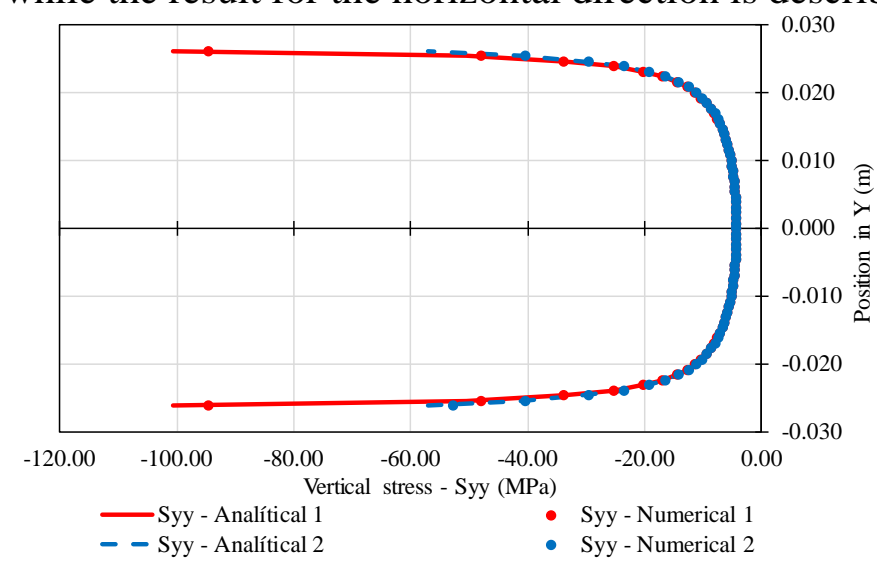

Figure 5: Vertical stress along the direction of the applied load 


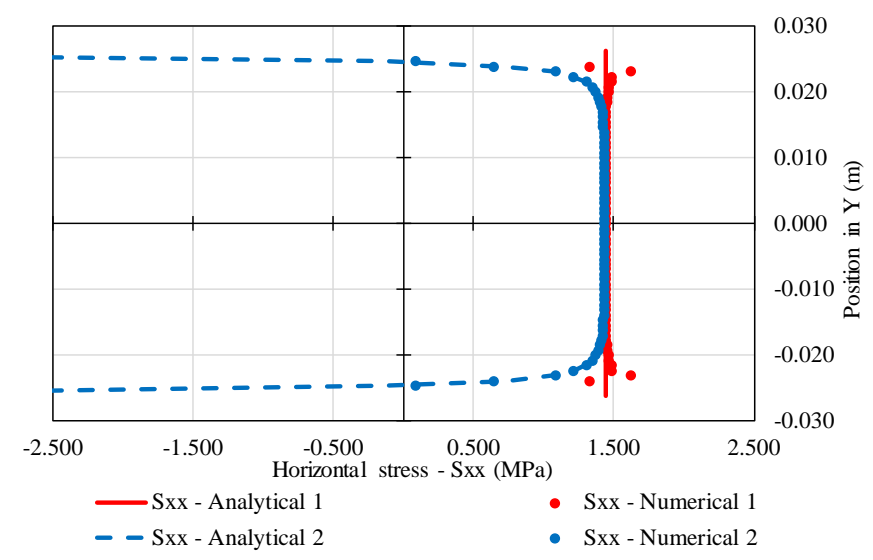

Figure 6: Vertical stress along the direction of the applied load

For the vertical stress, Model 1 and Model 2 are in agreement, as well as the numerical and analytical solutions. On the other hand, for the horizontal stress, Model 2 shows good agreement between analytical and numerical approaches while for Model 1 the results are quite different. In addition, the results from Model 1 and Model 2 are different in the horizontal stress.

The reason for the difference between the results from Analytical 1 and Numerical 1 is that the Analytical 1 considers a constant value in the horizontal stress along the direction of applied load. On the other hand, as Numerical 1 has a concentrated force, the stress field near the applied load changes because of stress concentration. From Figure 6, it is possible to conclude that when a concentrated load is applied to the sample, the maximum tensile stress is near the applied load, while for the distributed load the maximum tensile stress is in the middle of the sample.

To enhance the reliability of the DIC method, lab results are compared with the model with concentrated load (Model 1). Figure 7 displays the comparison between experimental (DIC), Analytical 1 and Numerical 1 results for the horizontal strain along the direction of applied load. Figure 8 exhibits the result for vertical strain.

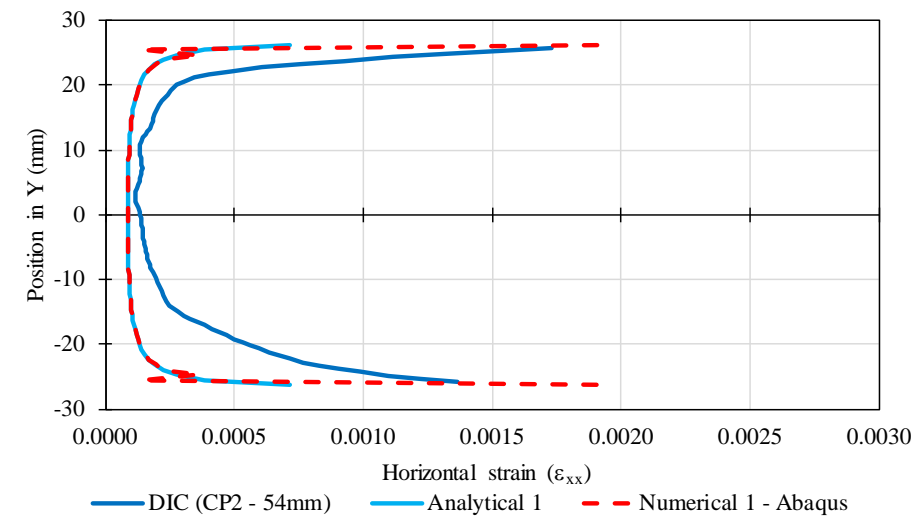

Figure 7: Horizontal strain along the direction of the applied load. 


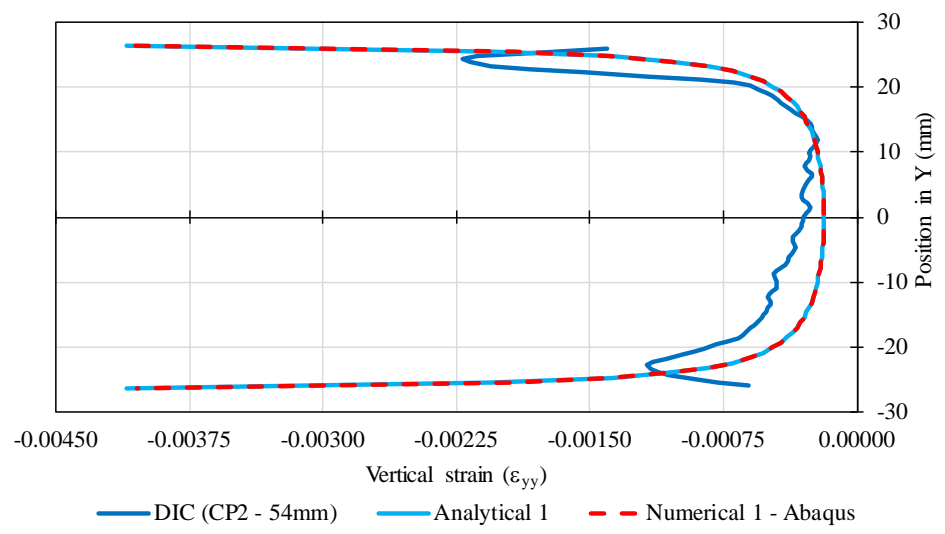

Figure 8: Vertical strain along the direction of the applied load.

It is observed from Figure 7 and Figure 8 that both Analytical 1 and Numerical 1 are in agreement with the laboratory results (DIC). This enhances the reliability of the DIC methodology applied in the Brazilian Splitting Test. In addition, it shows that the procedure used in the laboratory test is equivalent to applying a concentrated load in the sample, which would start the crack opening near the applied load.

\section{CONCLUSION}

The Brazilian Splitting Test was performed on four Indiana Limestone samples. DIC was adopted during the test to measure the development of displacement, and consequently, strain on these four samples. The stress and strain fields were also investigated by two numerical and two analytical solutions. By assessing the results from numerical and analytical models, good agreement was found. A difference is noticed when comparing the horizontal stresses near the point of applied load, as Model 1 considers a concentrated load, generating a stress concentration region, while Model 2 considers a distributed load.

When comparing Model 1 from analytical and numerical approaches with DIC, the results for the horizontal strain are very similar. This is due to the set-up used in the laboratory test. The piston was in direct contact with the sample, creating stress concentration near the point of applied load, as a concentrated load.

Another preliminary conclusion obtained from this work is regarding fracture initiation. When a concentrated load is applied on the sample, fracture tends to initiate near the point of applied load. This can be noticed by observing the high tensile strain near this point. On the other hand, fracture initiates close to the center of the sample when a distributed load is applied, as it presents a higher tensile strain around this point.

This study is currently under development, as it is important to confirm the numerical and analytical results by performing different laboratory tests, with set-ups capable of applying distributed load.

\section{REFERENCES}

[1] ASTM, "Standard Test Method for Splitting Tensile Strength of Intact Rock Core Specimens 1 D3967-08,” ASTM Int. West Conshohocken, PA, no. Reapproved, pp. 1-5, 2016.

[2] D. Li and L. N. Y. Wong, "The brazilian disc test for rock mechanics applications: Review and new insights,” Rock Mech. Rock Eng., vol. 46, no. 2, pp. 269-287, 2013.

[3] Y. Han, B. Lai, H.-H. Liu, and H. Li, "Measurement of elastic properties in Brazilian disc test: solution derivation and numerical verification," Geomech. Geophys. GeoEnergy Geo-Resources, vol. 4, no. 1, pp. 63-77, 2018. 
[4] X. Xu, S. Wu, Y. Gao, and M. Xu, "Effects of Micro-structure and Micro-parameters on Brazilian Tensile Strength Using Flat-Joint Model,” Rock Mech. Rock Eng., vol. 49, no. 9, pp. 3575-3595, 2016.

[5] Y. Ma and H. Huang, "Tensile strength calibration in DEM modeling," in American Rock Mechanics Association, 2017, p. 8.

[6] G. Walton, J. Arzúa, L. R. Alejano, and M. S. Diederichs, “A laboratory-testing-based study on the strength, deformability, and dilatancy of carbonate rocks at low confinement,” Rock Mech. Rock Eng., vol. 48, no. 3, pp. 941-958, 2015.

[7] Y. Jianhong, F. Q. Wu, and J. Z. Sun, "Estimation of the tensile elastic modulus using Brazilian disc by applying diametrically opposed concentrated loads," Int. J. Rock Mech. Min. Sci., vol. 46, no. 3, pp. 568-576, 2009. 